\title{
Circulating Th1, Th17 Cells and Related Cytokines: A Study in Psoriasis Patients in
}

\section{Bangladesh}

Sabia Shahin Sultana', Shirin Tarafder²*, Ismet Nigar ${ }^{3}$ and ATM Asaduzzaman ${ }^{4}$

'Department of Microbiology, Shaheed Suhrawardy Medical College, Dhaka, Bangladesh

${ }^{2}$ Department of Microbiology and Immunology, Bangabandhu Sheikh Mujib Medical University (BSMMU), Dhaka, Bangladesh

${ }^{3}$ Department of Microbiology and Immunology, Bangabandhu Sheikh Mujib Medical University (BSMMU), Dhaka, Bangladesh

${ }^{4}$ Department of Dermatology and Venereology, Bangabandhu Sheikh Mujib Medical University (BSMMU), Dhaka, Bangladesh

*Corresponding author: Shirin Tarafder, Professor, Department of Microbiology and Immunology, Bangabandhu Sheikh Mujib Medical University (BSMMU), Shahbag, Dhaka, Bangladesh

ORCID ID: https://orcid.org/0000-0001-6568-2864

\section{Abstract}

Introduction: Psoriasis is an autoimmune chronic inflammatory skin disease. Recent discovery of IL-23/Th17 axis focuses that alteration of cytokine mediated T cell populations are the main driver of disease severity and disease progression. The study aimed to evaluate role of Th1, Th17 cells and serum level of TNF- $\alpha$, IL-17 and IL-23 in psoriasis.

Materials and methods: This was a prospective type of cross sectional study. A total of 35 psoriasis patients and 35 healthy controls were enrolled. Disease severity was assessed by Psoriasis Area and Severity Index (PASI) scoring. Peripheral blood mononuclear cells were separated by Ficoll-Hypaque density centrifugation and circulating Th1 and Th17 cells were quantified by flow cytometric immuno-phenotyping. Serum TNF- $\alpha$, IL-17 and IL-23 level of study subjects were measured by ELISA.

Results: We observed frequency of both CD4+IFNY+/Th1 ( $P=0.001)$ and CD4+IL17+/Th17 $(P=<0.001)$ cells increased significantly in psoriatic patients when comparing with healthy controls. Th17 cells were increased with disease progression. We also observed higher concentration of serum TNF- $\alpha(P=0.002), I L-17(P=0.002)$ and IL-23 $(P=<0.001)$ in psoriasis patients. Frequencies of Th1 and Th17 cells and serum TNFa level were correlated with disease duration, whilst serum IL-17 level were correlated with disease severity. We didn't find any correlation of IL-23 with severity and disease duration.

Conclusion: Predominance of Th17 and Th1 cells along with elevated serum cytokines, is suggestive that IL-23/Th17 axis is activated in psoriasis. By evaluating these soluble biomarkers clinician can plan appropriate treatment for psoriasis patients and further disease progression can be halted accordingly.

Keywords: Th1 cell, Th17 cell, Immunophenotyping, IL-23/Th17 axis, Psoriasis Area and Severity Index

Introduction

Psoriasis is an immune mediated inflammatory skin disease which accounts for metabolic syndromes like obesity, insulin resistance, hypertension, dyslipidemia and atherosclerosis. In past years psoriasis was characterized by increased production of Th1 cells in response to YIFN. Recent discovery of IL-23/ Th17 axis has broken the long- held paradigm regarding the role of other $T$ cell lineages and associated cytokines [1, 2].

In response to different cytokine (IL-6, IL-1 and TGF $\beta$ ) host protective Th17 cells are produced. These cells are mainly restricted to the gastrointestinal tract and found to be low or in undetectable condition in serum. 
They promote mucosal defense, barrier tissue integrity and wound healing. Whereas IL-23 induced pathogenic Th17 cells promote chronic tissue inflammation during infection, granuloma formation and autoimmunity [1, 3]. It has been hypothesized that in genetically susceptible person, psoriasis auto-antigens (LL-37/cathelicidin, ADAMTSL5 and PLA2G4D) are produced in response to different stimulatory factors (bacterial/ viral infection, trauma). Upon presentation of these antigens epidermal plasmacytoid dendritic cells (pDCs) of pre-psoriatic skin release TNF- $\alpha$ which in turn activated myeloid dendritic cells (mDCs). Activated mDCs then migrate into draining lymph nodes and drive differentiation into IL-17 secreting highly pathogenic Th17 cells by secreting IL-23. These highly inflammatory Th17 cells act on keratinocytes via production of IL-17A, IL-17F, IL-22 and TNF- $\alpha$. Absence of this Th17 cells in normal skin indicates its prime role in psoriasis. Hence IL-23 called the master regulator of IL-23/ Th17 axis, which is a heterodymic cytokine of IL-12 family consisting of a p19 subunit and a p40 subunit that is shared with IL-12 [1,4].

In presence of IL-23, IL-17 can also be secreted from CD8+ $T$ cells (Tc17), innate lymphoid cells (ILCs) and $\gamma \delta$ $T$ cells in skin. Primary effects of IL-17 on keratinocytes include indirect induction of epidermal hyperplasia through IL-19 and IL-36 and up regulation of the innate immune response and antimicrobial peptides (eg, hBD2, S100A7, and LL-37). Through increased production of keratinocytederived chemokine, IL-17 also recruits neutrophils and mDCs in epidermis. It also causes transcription of multiple pro-inflammatory genes (eg: IL-1 $\beta, \mathrm{IL}-6$, and IL-8) that act synergistically with TNF- $\alpha$ to sustain the inflammatory events in psoriatic skin. IL-17 also attracts Th1 cells into psoriatic skin. Once activated, human Th17 cells may remain as effector resident memory cells for extended periods and have been identified in previously lesional psoriatic skin even after clinical resolution. Increased mRNA levels of the IL-23/Th17 axis, including IL-23p19, IL-12/23p40, IL-22, IL-17A and IL-17F in psoriatic skin underscore their role in disease pathogenesis and progression (4-6).

Supporting the evidence anti IL-17 monoclonal antibodies (mAbs) show good clinical response in psoriasis. Clinical trial data for ustekinumab, a selective mAbs antagonist $\mathrm{p} 40$ subunit focuses the central role of cytokines as predominant drivers of psoriatic disease. As a "master regulator" of Th17 cell development, IL-23 inhibition targeting $\mathrm{p} 19$ subunit are now the new therapeutic solutions. In early trial IL-23p19 antagonists also showed long time treatment response with just a single dose [4]. These novel therapeutic agents are now used widely throughout the world due to its potential modification in clinical outcome with limited side effects. But in Bangladesh use of biologics in psoriasis is limiting due to unavailable data regarding it.

Since etiology of psoriasis is complex and driven primarily by an aberrant immune response in the skin, we aimed to demonstrate the circulatory Th17 and Th1 cells and related serum cytokines to assess their role in psoriasis. This study can provide an insight cellular picture of disease status by focusing their role in severity and disease duration and help the clinician of our country to choose novel potential therapeutic options like biologics.

\section{Materials and Method}

\section{Ethical approval}

This study was carried out in the department of Microbiology and Immunology, Bangabandhu Sheikh Mujib Medical University (BSMMU), Dhaka, Bangladesh from March 2019 to February 2020. This study was approved by institutional review board (IRB) of BSMMU (BSMMU/2019/6913) and all subjects provided informed consent for study and publication.

\section{Subjects}

A total of 35 psoriatic patients and 35 healthy controls were enrolled according to the Declaration of Helsinki in this cross-sectional study. Psoriasis patients were diagnosed by an expert dermatologist. Psoriasis patient with diabetes, infection, pre-existing thyroid disease, hypertension, malignancies and undergoing systemic therapy in the last three months and topical treatment for 1 month were excluded. Individuals, without any skin and infectious diseases and without a family history of autoimmune diseases, were recruited as healthy controls [7]. The clinical characteristics including disease severity (assessed by psoriasis area and severity index, PASI scoring) [8], disease duration, past therapies, associated comorbidities and the detailed family history were recorded. Six $\mathrm{ml}$ of peripheral venous blood was collected from each study subject. $3 \mathrm{ml}$ blood taken in heparinized tube for immuno-phenotyping and $3 \mathrm{ml}$ taken in tube without anticoagulant, then centrifuged at $4000 \mathrm{rpm}$ for 5 minutes. Separated serum was stored at $-20^{\circ} \mathrm{C}$ till analysis of cytokines.

\section{Methods}

Antibodies: Different conjugated antibodies were used. FITC conjugated anti-CD3 (UCHT1) was obtained from Abcam, UK. PC5 conjugated anti-CD4 (13B8.2) and ECD conjugated anti-CD45 (J33) were obtained from BECKMAN COULTAR. PE conjugated anti-IFNY (HIY3a) and anti-IL17 (SCPL1362) were obtained from BD Biosciences. 
Cell isolation and flow cytometric analysis of Th17 and Th1 cells: Heparin containing blood samples were diluted 1:2 with phosphate buffer saline and peripheral blood mononuclear cells (PBMCs) were isolated using density gradient centrifugation on Ficoll Hypaque (Invitrogen, Germany) and adjusted at a concentration of $106 / \mathrm{ml}$ in RPMI 1640 medium (Invitrogen, Germany). Then PBMCs were stimulated by 50 ng Phorbal-12-myristate-13-acetate/ PMA for 5 hours at $37^{\circ} \mathrm{C}$ in the presence of $1 \mu \mathrm{l} \mathrm{Golgi}$ plug protein transport inhibitor. To prevent nonspecific binding of monoclonal antibodies, $1 \mu \mathrm{g}$ BD Fc block added in cell media. Cells were then centrifuged, washed and resuspended in $50 \mu \mathrm{l}$ staining buffer ( $1 \%$ fetal bovine serum and $0.09 \%$ sodium azide solution in phosphate-buffered saline). Then cells were incubated with $20 \mu$ l of each cell surface monoclonal antibody CD3 - FITC, CD4-PC5 and CD45- ECD for 30 minutes at $4^{\circ} \mathrm{C}$. After that cell were washed twice, fixed and permeabilized by Cytofix/CytopermTM Plus (BD Biosciences). Following that cell were again washed twice and pellet and then separated in two tube. Then 20 $\mu \mathrm{l}$ intracellular antibodies for IFNY-PE and IL-17- PE were added in tube 1 and tube 2 respectively and incubated for 30 minutes at $4^{\circ} \mathrm{C}$. Tube 1 represents $C D 4+I F N Y+/ T h 1$ cell and Tube 2 represents CD4+IL-17+/Th17 cell. Flow cytometry was performed in BECKMAN COULTER CYTOMICS FC 500. Minimum 10,000 events were taken for interpretation.

Detection of serum level of TNF- $\alpha$, IL-17 and IL-23 by Enzyme Linked Immunosorbent Assay (ELISA): Frozen serum was thawed and the level of TNF- $\alpha$, IL-17 and IL-23 were measured by ELISA kit (Ray-Biotech, USA; Catalog \#: ELH-TNFa, Catalog \#: ELH-IL17 and Catalog \#: ELH-IL23) as per standard protocol following manufacturer's instruction. According to principle, all three tests were quantitative sandwich ELISA. The values were recorded at a wavelength of $450 \mathrm{~nm}$. Standard curve was generated for each cytokine by plotting the average absorbance of each standard on vertical axis versus the corresponding cytokine standard concentration on the horizontal axis. The number of cytokines in each sample were determined by extrapolating OD values against cytokine standard concentration using the standard curve.

\section{Statistical Analysis}

Quantitative data were expressed as mean \pm SD. To compare differences of $\mathrm{T}$ cell subsets, independent sample $t$ test was used. Comparison of serum cytokines between groups were done by Mann-Whitney-U-test. Correlations were assessed using the Pearson correlation test. For all test a $P$ value $<0.05$ was considered as statistically significant. Statistical analyses were performed using SPSS software package version-22 (Strata Corporation, College station,
Texas).

Results

Increased circulating Th1 and Th17 cell frequencies in peripheral blood of psoriasis patients correlated with disease duration

We first analyzed expression of IFNY and IL-17 in CD4+ $T$ cell proportions in psoriatic patients and then compare with that of healthy controls. Frequency of circulating CD4+ IFNY+/Th1 cells were significantly higher in psoriasis patients ( $2.79 \pm 2.07$ Vs $1.43 \pm 0.42, \mathrm{P}=0.001)$ (Figure $1 \mathrm{~A}$, Supplementary Table 1). CD4+IL17+/Th17 cells were also significantly higher in psoriasis patients $(1.80 \pm 1.56$ Vs 0.23 $\pm 0.20, P<0.001$ ) (Figure $1 \mathrm{~B}$, Table Supplementary Table 1). Furthermore, we analyzed the association between CD4+ IFNY+/Th1 and CD4+IL17+/Th17 cells with disease severity and disease duration. Both cells were positively correlated with disease duration and inversely correlated with PASI (Figure 2A and 2B, Supplementary Table 2). We also observed Th1 cells were predominant in acute stage of severe psoriasis patients (Figure $3 \mathrm{~B}$ and $3 \mathrm{C}$ ), while Th17 cells tends to increase with disease progression (Figure 3D and $3 E$ ). These data highlight the self-amplified $T$ cell responses in chronic inflammatory events of psoriasis.

\section{Serum TNF- $\alpha$, IL-17 and IL-23 levels are elevated in psoriasis}

We observed significant increased level of serum TNF- $\alpha$ in psoriasis patients ( $534.68 \mathrm{pg} / \mathrm{ml} \mathrm{Vs} 84.26 \mathrm{pg} / \mathrm{ml}, \mathrm{P}=0.002)$. Interestingly serum levels of TNF- $\alpha$ positively correlated with disease duration but inversely correlated with PASI (Figure 2C, Supplementary Table 2). Serum IL-17 levels were also significantly increased in psoriasis patients (150 $\mathrm{pg} / \mathrm{ml} \mathrm{Vs} 84.10 \mathrm{pg} / \mathrm{ml}, \mathrm{P}=0.002$ ) and positively correlated Supplementary Table 1: Circulating Th1 and Th17 cell subsets in peripheral blood in study population, Related to Figure 1.

\begin{tabular}{|c|c|c|c|}
\hline $\begin{array}{c}\text { T cell } \\
\text { subtypes }\end{array}$ & $\begin{array}{c}\text { Patients } \\
(n=31) \\
\text { Mean of } \% \pm S D \\
\text { (range) }\end{array}$ & $\begin{array}{l}\text { Healthy Controls } \\
\qquad(n=27) \\
\text { Mean of } \% \pm S D \\
\text { (range) }\end{array}$ & $P$ value \\
\hline $\begin{array}{c}\text { Th1 } \\
\left(\mathrm{CD} 4^{+} \mathrm{IFNY}^{+}\right)\end{array}$ & $\begin{array}{c}3.30 \pm 2.59 \\
(0.50-11.40)\end{array}$ & $\begin{array}{l}1.31 \pm 1.25 \\
(0.1-4.40)\end{array}$ & 0.001 \\
\hline $\begin{array}{c}\text { Th17 } \\
\left(\mathrm{CD} 4^{+} \mathrm{IL} 17^{+}\right)\end{array}$ & $\begin{array}{c}1.80 \pm 1.56 \\
(0.50-7.40)\end{array}$ & $\begin{array}{c}0.23 \pm 0.20 \\
(0.10-1.00)\end{array}$ & $<0.001$ \\
\hline
\end{tabular}

Note: $P$ value was calculated by an independent sample $t$ test.

Cell Percentage (\%) are from CD3+CD4+T lymphocytes.

Samples of 4 patients and 8 healthy controls were discarded due to lack of their quality. 
Supplementary Table 2: Correlation of PASI and disease duration of psoriasis patients with circulating $T$ helper cells and cytokines, Related to Figure 2.

\begin{tabular}{|c|c|c|c|c|}
\hline & \multicolumn{2}{|c|}{ PASI } & \multicolumn{2}{c|}{ Duration of diseases } \\
\hline & r value & P value & r value & P value \\
\hline Th-1 cell & -0.26 & 0.12 & 0.18 & 0.29 \\
\hline $\begin{array}{c}\text { Th-17 } \\
\text { cell }\end{array}$ & -0.18 & 0.92 & 0.17 & 0.34 \\
\hline TNF- $\alpha$ & -0.1 & 0.56 & 0.32 & 0.32 \\
\hline IL-17 & 0.08 & 0.64 & -0.78 & 0.66 \\
\hline IL-23 & -0.05 & 0.76 & -0.09 & 0.63 \\
\hline
\end{tabular}

Note: Correlation were assessed by Pearson correlation test. " $r$ " value without any sign indicates positive correlation and " "indicates inverse correlation. Value > 0.7-1 indicates strong correlation. inflammatory markers by $\mathrm{T}$ cells along with multifactorial genetic factor play a key role in pathogenesis of psoriasis. To see the status of $\mathrm{T}$ helper cells in chronic inflammatory events in psoriasis, we first analyze CD4+ IFN $Y+$ Th1 and CD4+IL17+ Th17 cells in peripheral blood of psoriasis patients. We observed significant elevation in the percentage of Th1 cells (CD4+IFNY+) in psoriatics. In a previous study done in India based on whole blood flowcytometric assay showed increase IFN $Y$ expression in CD4+ lymphocytes in psoriasis patients compared to healthy controls [7]. Studies done in Hungary and USA show similar findings $[9,10]$. Though in this study, a difference in median value between psoriatics and healthy controls was seen with a correlation with disease duration, Th1 cells were not correlated with disease severity. In consistent with this, another study showed significantly higher level of circulating Th1 cells
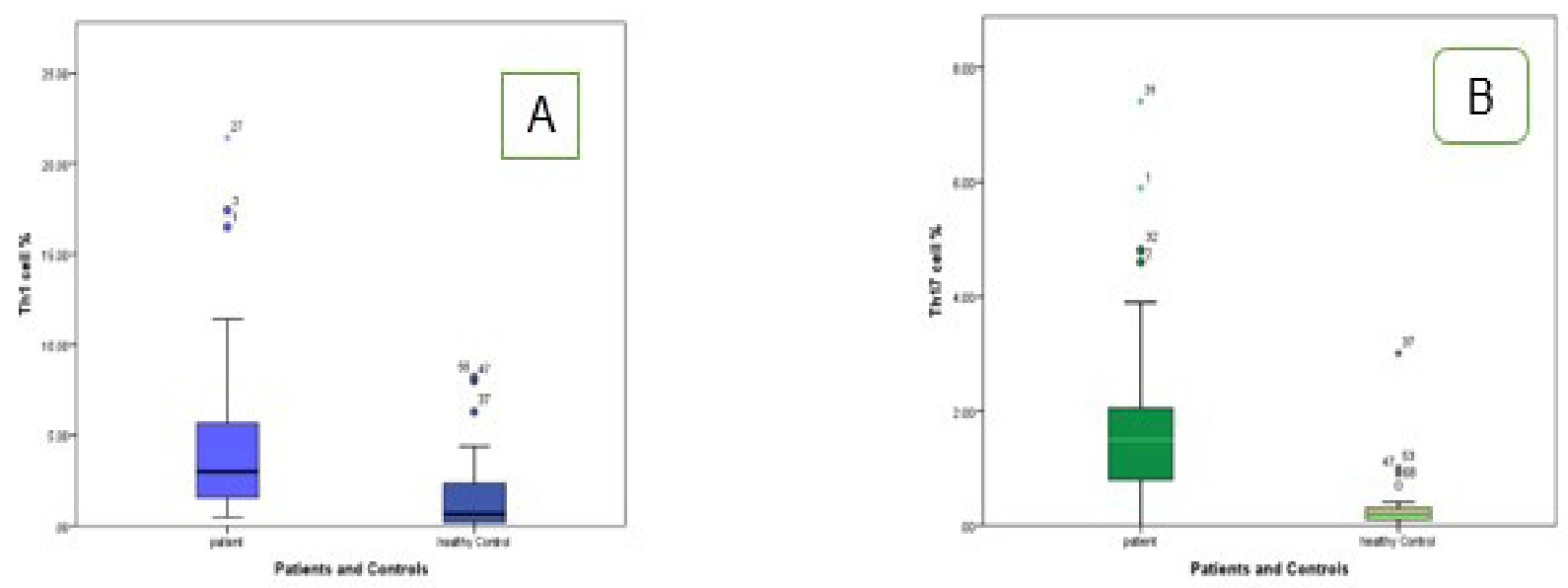

Figure 1: Comparison of percentage of circulating Th1 $(C D 4+I F N Y+)$ and Th17 $(C D 4+I L 17+)$ cells in peripheral blood of study population (A) CD4+ IFNY+ Th1 cells (B) CD4+IL17+ Th17 cells. Dark line within each box indicates the median value. Fifty percent of the data lied inside the box. Line/whisker extended from box indicates distribution of data. Indicators along this line with number indicates outliers (abnormally distributed data.

with PASI (Figure 2D, Supplementary Table 2). In addition, we did not find any mutual correlation between Th17 cell and IL-17 in psoriasis patients (Figure 4). Though serum IL23 levels were significantly increased in psoriasis patients ( $231.70 \mathrm{pg} / \mathrm{ml}$ Vs $13.97 \mathrm{pg} / \mathrm{ml}, \mathrm{P}=<0.001$ ) but no correlation was observed with PASI and with disease duration (Figure $2 \mathrm{E}$, Supplementary Table 2). These data signify the role of IL-17 and IL-23 in disease pathogenesis. It also implies the fact that TNF- $\alpha$, a potent inflammatory cytokine tends to increase as disease progresses. Thus, providing the fact that clinical assessment should be correlated to judge the actual cellular status of a psoriasis patient.

\section{Discussion}

Psoriasis is now thought to be a T helper cell mediated autoimmune disease. Altered production of different
$(2.79 \pm 2.07$ Vs $1.43 \pm 0.42, P<0.01)$, and were not correlated with disease severity [11]. We also observed Thus this study provides evidence of an imbalance towards the Th1 response in psoriatic patients. It may be due to the fact that T helper cells shows plasticity and co-expression of different surface phenotype.

It has been demonstrated that IL-17 producing CD4+ Th17 cells mediate inflammation in psoriasis and psoriatic arthritis and other autoimmune diseases such as rheumatoid arthritis and ankylosing spondylitis [12]. In this study, frequency of circulating Th17 cells (CD4+IL17+) observed in psoriatic patient were significantly higher in comparison to healthy controls. It showed correlation with disease duration $(r=0.17, P=0.34)$ but not with PASI $(r=-0.18, P=0.92)$. Similar findings have been observed in several studies. A previous

Citation: Sabia Shahin Sultana ${ }^{1}$, Shirin Tarafder ${ }^{2}$, Ismet Nigar ${ }^{3}$ and ATM Asaduzzaman ${ }^{4}$. Circulating Th1, Th17 Cells and Related Cytokines: A Study in Psoriasis Patients in Bangladesh

Op Acc J Bio Sci \& Res 9(1)-2021 
A
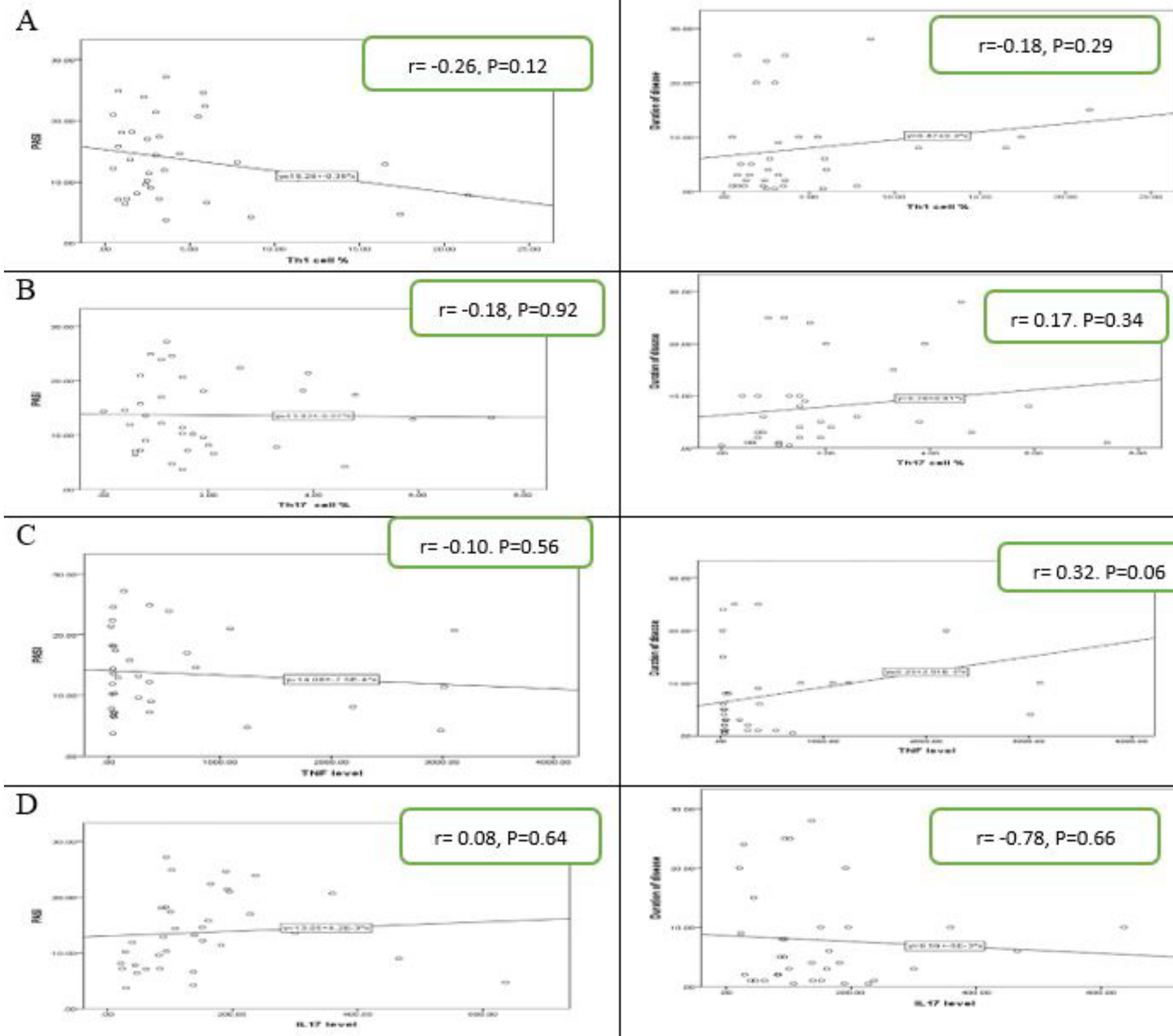

(1)
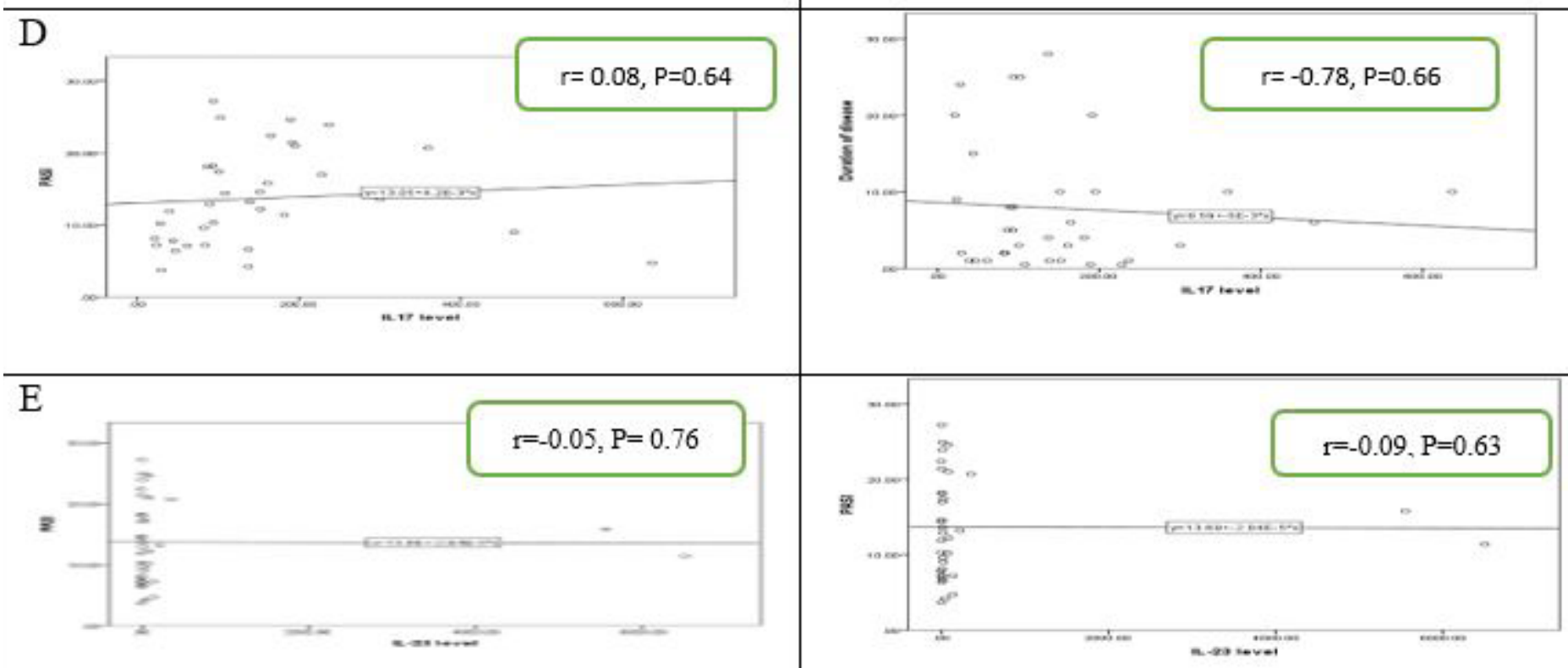

Figure 2: Correlation circulating Th cells and serum cytokines with PASI and disease duration in psoriasis patients. $(A, B, C) C D 4+\gamma I F N+T h 1$ cells, CD4+IL17+Th17 cells and serum TNFa level positively correlated with disease duration and inversely correlated with PASI. (D) Serum IL-17 level positively correlated with PASI but inversely correlated with disease duration. Correlation were assessed by Pearson correlation test. $r$ value without any sign indicates positive correlation and "-" indicates inverse correlation. Value $>0.7-1$ indicates strong correlation.

Citation: Sabia Shahin Sultana ${ }^{1}$, Shirin Tarafder ${ }^{2 *}$, Ismet Nigar ${ }^{3}$ and ATM Asaduzzaman ${ }^{4}$. Circulating Th1, Th17 Cells and Related Cytokines: A Study in Psoriasis Patients in Bangladesh

Op Acc J Bio Sci \& Res 9(1)-2021 
A

\section{Healthy control}

B

Psoriasis patient

PASI 6.4

disease duration-

1year
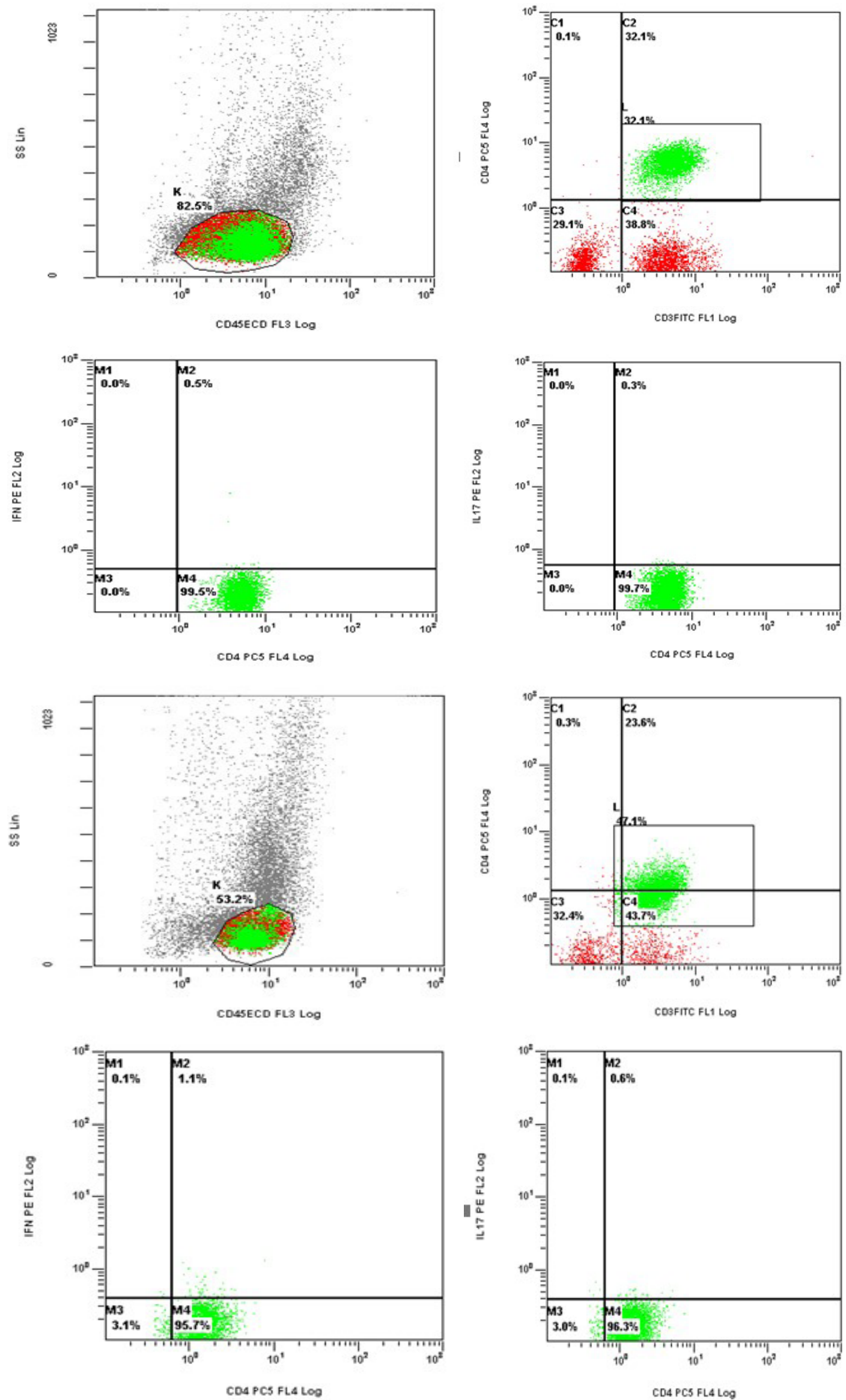

Citation: Sabia Shahin Sultana ${ }^{1}$, Shirin Tarafder², Ismet Nigar ${ }^{3}$ and ATM Asaduzzaman ${ }^{4}$. Circulating Th1, Th17 Cells and Related Cytokines: A Study in Psoriasis Patients in Bangladesh

Op Acc J Bio Sci \& Res 9(1)-2021 
C

Psoriasis patient

PASI 4.7

disease duration10years

D

Psoriasis patient

PASI 24.6

disease duration6month

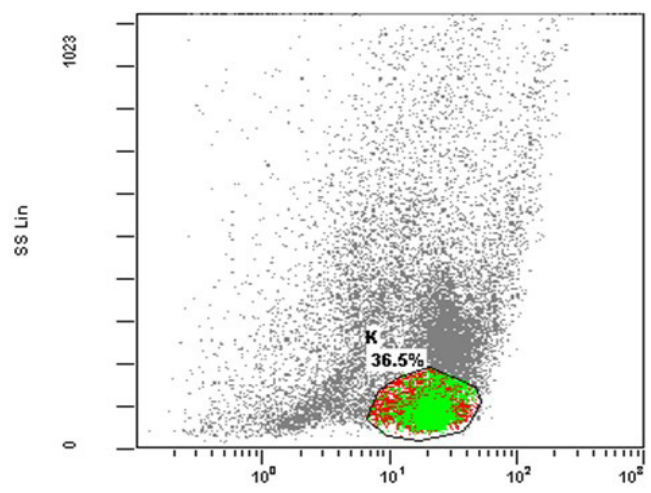

CD45ECD FL3 Log

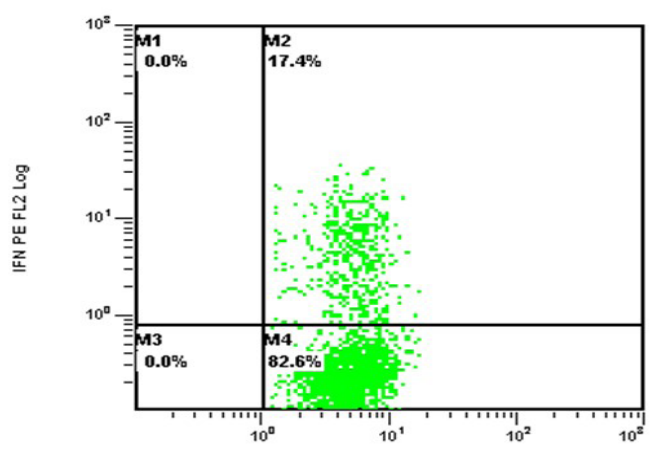

CD4 PC5 FL4 Log
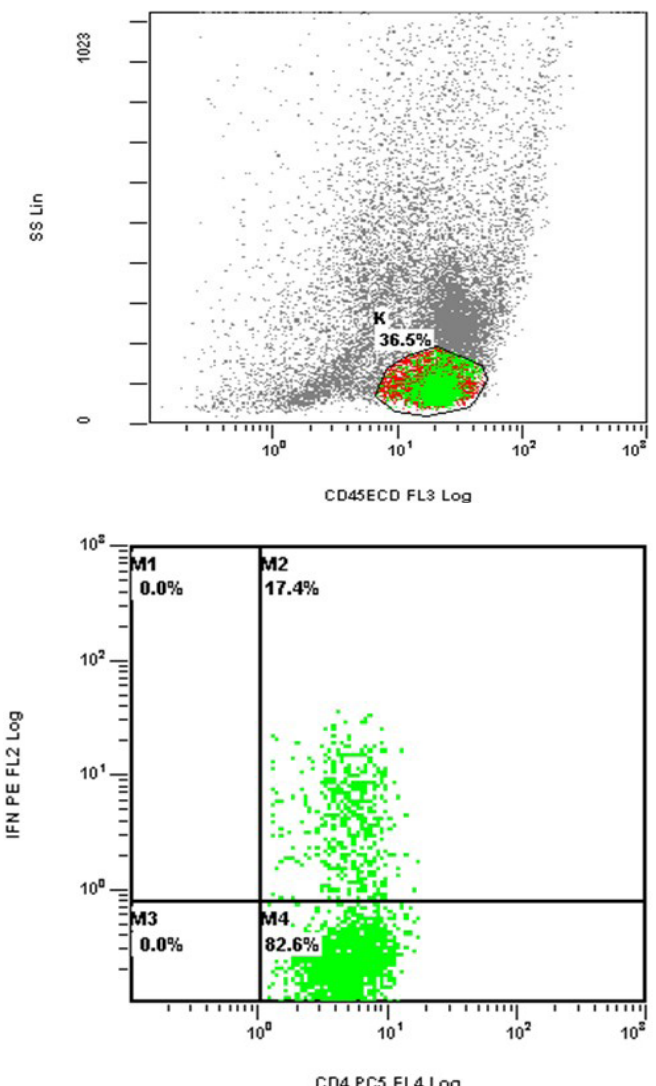

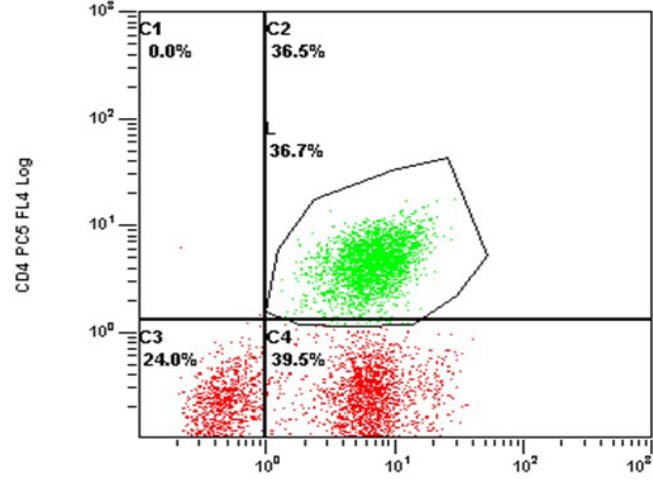

CD3FITC FL1 Log
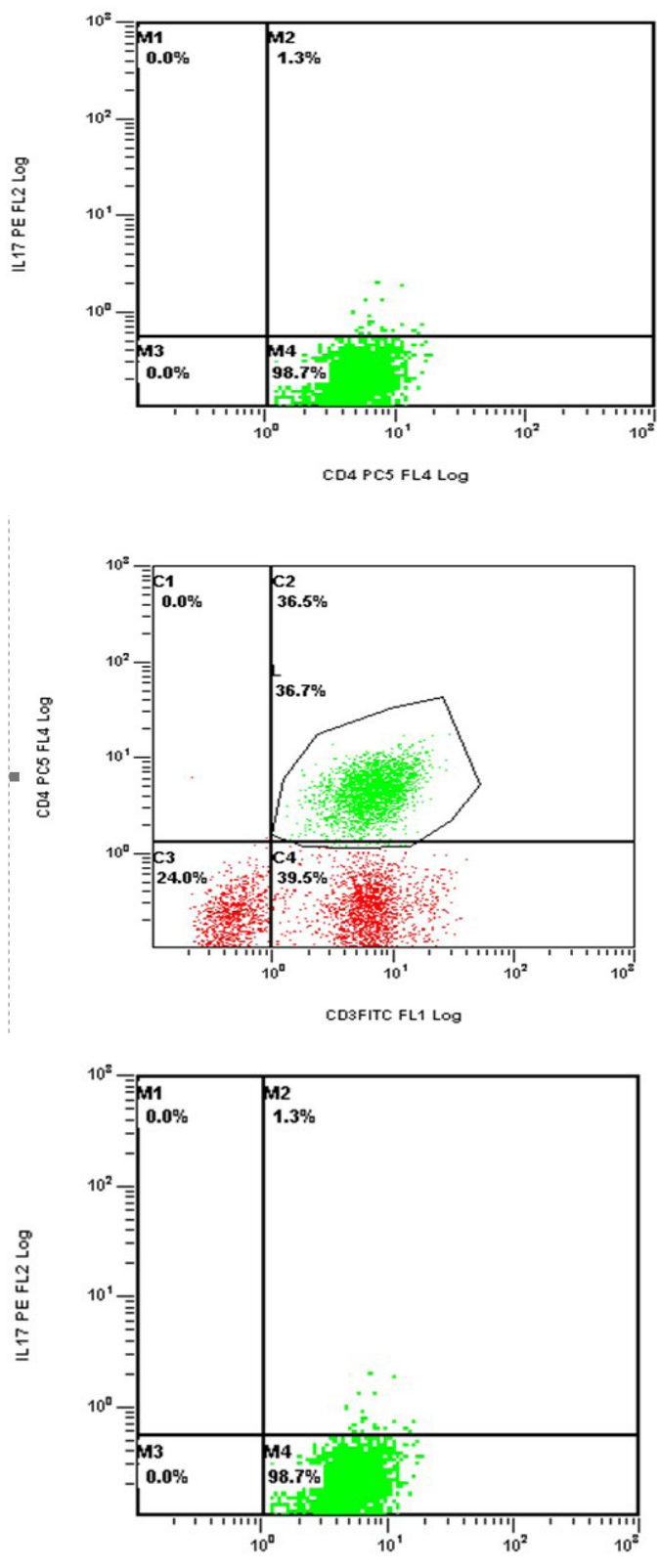

CD4 PC5 FL4 Log

Citation: Sabia Shahin Sultana ${ }^{1}$, Shirin Tarafder ${ }^{2}$, Ismet Nigar ${ }^{3}$ and ATM Asaduzzaman ${ }^{4}$. Circulating Th1, Th17 Cells and Related Cytokines: A Study in Psoriasis Patients in Bangladesh 


\section{$\mathbf{E}$}

\section{Psoriasis patient \\ PASI 27.2} disease duration25years
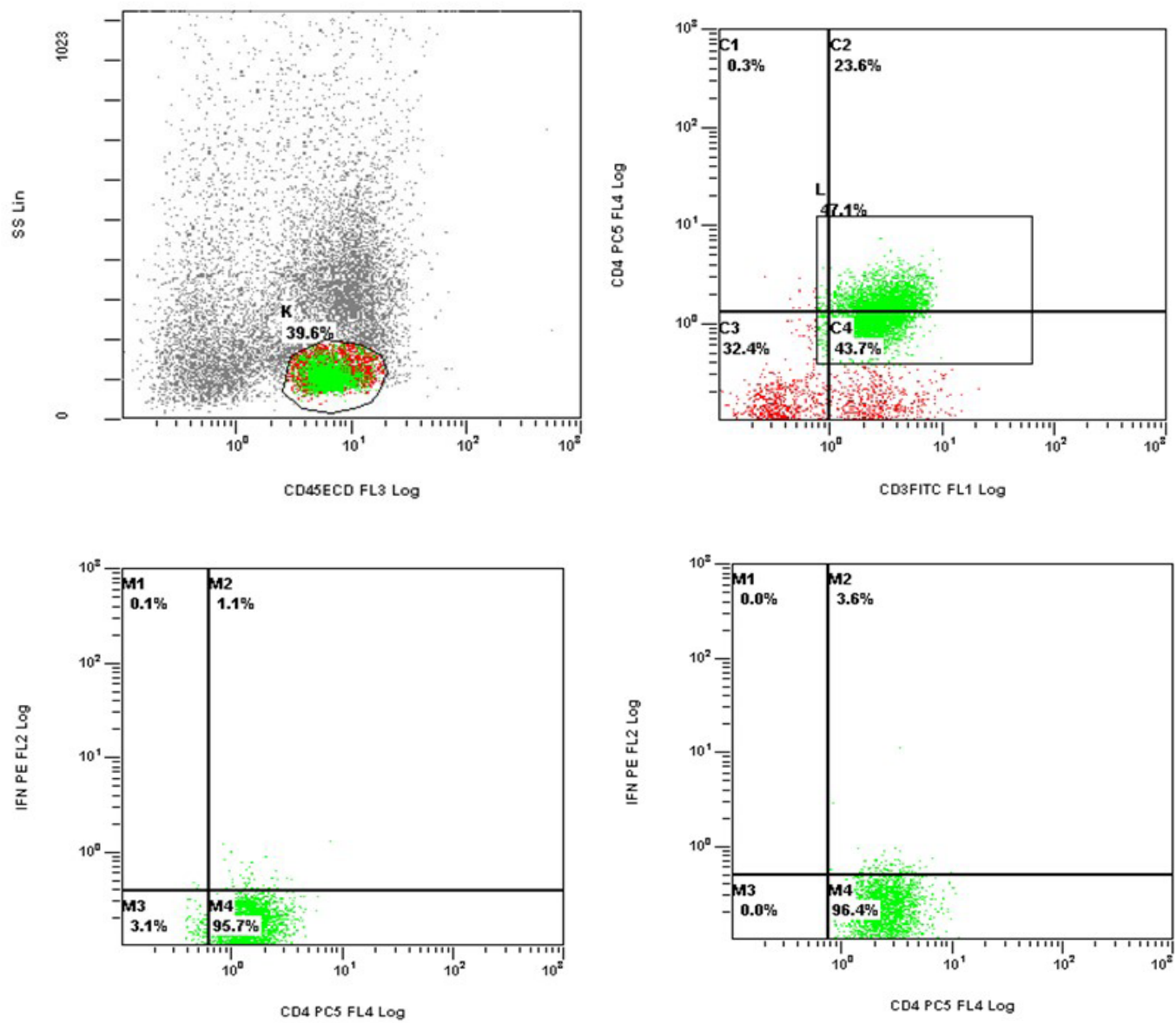

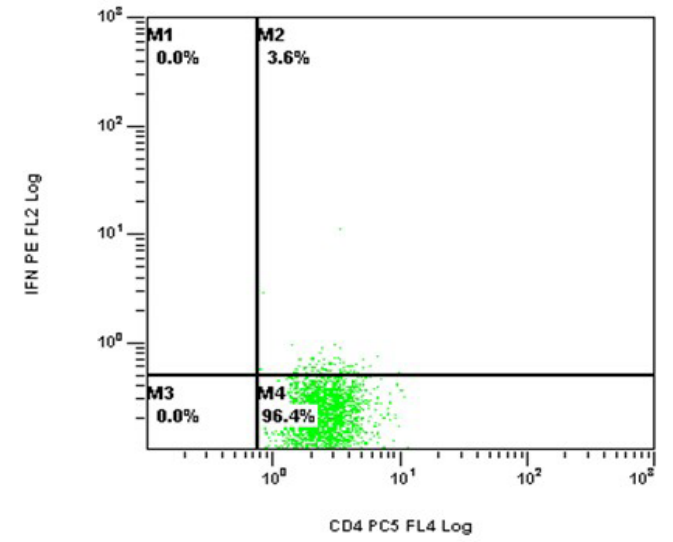

Figure 3: Th1 and Th17 cells increases with disease duration in psoriasis. PBMCs were stimulated and gated on lymphocytes (forward Vs side light scatter). Ascertained CD4+CD3+ cells were further sub gated to see expression of IFNY and IL-17. CD4+ IFNY+ cells represents Th1 cells and CD4+IL17+ cells represents Th17 cells. In psoriasis patient both Th1 and Th17 cells were increased than healthy controls (A). In mild psoriasis patient Th1 and Th17 cells increases with disease duration. (B and C). In acute stage of severe psoriasis Th1 cells were predominant, while with disease progression Th17 cells tends to increase (D and E).

study done in USA showed similar results where frequency of circulating Th17 cells were $1.47 \pm 0.74$ in psoriatics and $0.73 \pm 0.34 \%$ in healthy controls ( $P$ value of $<0.001$ ) but it was not correlated with disease severity [10]. Another study in China by Zhang et al. showed that frequency of circulating Th17 cells was elevated both in peripheral blood and in skin in psoriatics and were correlated with disease severity [13]. Similar observation was found in other studies $[7,14]$. Th17 cells were significantly elevated with a high median value in this study, but the correlation was not significant. It is may be due to small sample size $(n=35)$ and low PASI score $(<28)$ in comparison with other studies who found significant correlation. Aforementioned findings demonstrate the critical role of Th17 cells in disease pathogenesis of psoriasis.

Interestingly we observed, both Th 1 and Th17 cells increased in mild psoriatics. But in severe cases Th17 cells remain predominant with chronic cases whilst Th1 in earlier state. These can be explained by $T$ cell plasticity. Zhang et al. demonstrate that increased Th17 cells are accompanied with FoxP3+ Treg cell in psoriasis [13]. These Treg cells gradually loss its regulatory activity and become IL17+ cell and remain as one of the prime driver of local chronic inflammatory events [15]. This finding is also supportive of the autoimmune disease model of psoriasis.

Further we also measured serum levels TNF- $\alpha$, IL-17 and IL-23 to see association with disease pathogenesis that might reflect the activity of the cells usually present in the psoriatic lesions, in the active stage of the disease. In this study serum level of TNF- $\alpha$ was significantly elevated in psoriatics compared with healthy controls with a weak correlation with disease duration. In a study conducted in Greece, showed serum levels of TNF- $\alpha$ were significantly higher in psoriatic patients compared to those of controls $(P<0.01)$ without any significant difference between the groups. Also they didn't find any correlations with PASI [16]. Elevated level of TNF- $\alpha$ also been reported in many studies in

Citation: Sabia Shahin Sultana ${ }^{1}$, Shirin Tarafder ${ }^{2}$, Ismet Nigar ${ }^{3}$ and ATM Asaduzzaman ${ }^{4}$. Circulating Th1, Th17 Cells and Related Cytokines: A Study in Psoriasis Patients in Bangladesh

Op Acc J Bio Sci \& Res 9(1)-2021 


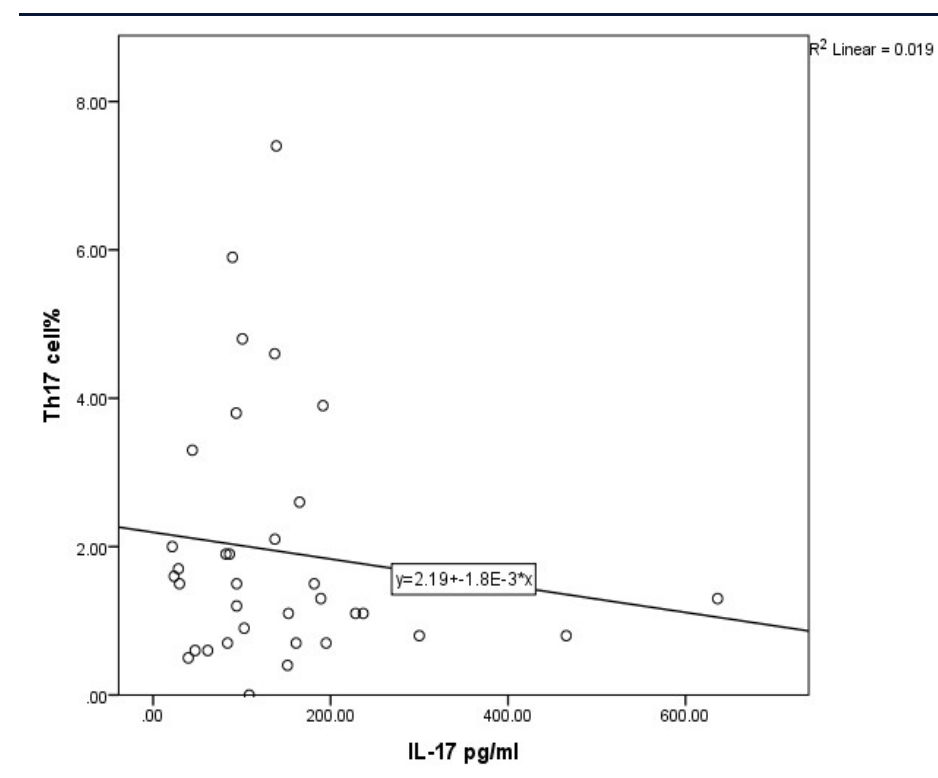

Figure 4: Correlation between IL-17 and Th17 cells in psoriasis patients. Cells were obtained from psoriasis patients and PBMCs were purified. Serum level of IL-17 level was inversely correlated with Th17 cells in psoriasis patients. Pearson correlation was done to see the level of significance ( $r$ value without any sign indicates positive correlation and "- " indicates inverse correlation. Value > 0.7- 1 indicate strong correlation).

patients with active psoriasis with positive correlation with PASI $[17,18]$. Several studies observe association of TNF- $\alpha$ in psoriatics by applying anti TNF- $\alpha$ monoclonal antibody therapy like infliximab and found decreased level of TNF- $\alpha$ after therapy [19]. It underscore pathogenic role of TNF- $\alpha$ in psoriasis. The present study showed concordance and discordance with the findings of aforementioned studies regarding association of TNF- $\alpha$ with disease severity.

IL-17 is a potent inflammatory cytokine, down regulate the T-regulatory cell activity and promotes Th1 cell differentiation [7]. Several studies reported increased mRNA levels of the IL-23/Th17 axis in psoriatic lesions. In present study both IL-17 and IL-23 levels were elevated in psoriasis patients in respect to healthy controls. Only IL-17 was weakly correlated with disease severity. We did not observe any correlation between serum level of IL-17 and Th17 cells in this study.

The findings of several studies are in agreement with the present study. The studies in China and Japan found significant serum level of IL-17 along with strong correlation with disease severity $[4,20]$. A study in Brazil showed increased IL-17 level in psoriasis patients in comparison with healthy controls. They also observed differences among patients with different degree of severity, but no statistical correlation with severity was found [21]. Another study done in Libya showed, increased IL-17 in psoriatics with no correlation with PASI [22]. A study in India showed, the mean plasma levels of IL-23 was significantly increased in psoriasis patients, compared with that of controls ( 37.65 \pm 19.4 vs. $34.55 \pm 21 \mathrm{pg} / \mathrm{mL}, p=0.02$ ) [23]. Chhabra et al. didn't found any significant difference and correlation in serum IL-17 and IL-23 level between psoriasis patients and healthy controls [24]. Different measurement methods other than ELISA, racial differences, larger study population and wide range of PASI may attribute discrepancies in different studies. IL-23 has been demonstrated to be a key cytokine in the inflammation in peripheral tissues and IL-17 is produced by Type 17 cells including CD4+ T cells (Th17), CD8+ T cells (Tc17), type- 3 innate lymphoid cells (ILCs) and $\gamma \delta$ T cells. But activated Th17 cells are the major source of IL-17 during inflammation. Production of IL-17 by these cells is influenced by mDCs derived IL-23 $[4,25]$. So, it can be concluded that elevation of serum IL-17 and IL-23 level in psoriatics are consequences of immune dysregulation.

Despite some conflicting data, it appears to be consensus that circulating Th1 and Th17 cells along with serum TNF- $\alpha$, IL-17 and IL-23 are increased in psoriatics. Being a master regulator IL-23 promotes early differerntiation and survival of pathologic Th17 cells. Activated Th17 cells secrets proinflammatory cytokine IL-17 and also IL-22, IL-21 and TNF- $\alpha$ which further contribute to the development of psoriatic plaques. So, by targeting IL-23 overall number of pathogenic Th17 cells can be reduced over time, which may also have a better long-term control on psoriasis. Kagami et al. observed greater decreasing trend in Th17 cell numbers and clinical parameters when compared to Th1 cell numbers in patients treated with anti TNF- $\alpha$ monoclonal antibody therapy, Infliximab [10]. Anti-IL-17 mAbs secukinumab, ixekizumab and brodalumab are also used to treat moderate to severe psoriasis. Also, IL-23 p40 inhibitor ustekinumab is being suscessfully used in psoriasis since 2009. As expected, drugs targeting P19 subunit of IL-23 shows a long-time treatment response in clinical trials implying the role IL-23 as predominant driver of psoriasis. Focusing on that FDA approved anti IL-23 monoclonal antibody guselkumab, risankizumab, and tildrakizumab for effective treatment of moderate to severe psoriasis in recent years [26-28].

It has also been reported that Th17 cells in psoriasis patients may contribute to skin disease and inflammation at sites other than skin. Elevated serum IL-17 and TNF- $\alpha$ levels are related with left ventricular diastolic dysfunction, heart diseases, hypertension, type 2 diabetes as well as metabolic syndrome $[29,30]$. These cytokines are now considerate as biomarker of metabolic diseases. Interestingly we observed an increasing trend of Th17 cells and serum TNF- $\alpha$ along with disease duration in psoriatics, which can be correlated

Citation: Sabia Shahin Sultana ${ }^{1}$, Shirin Tarafder ${ }^{2 *}$, Ismet Nigar ${ }^{3}$ and ATM Asaduzzaman ${ }^{4}$. Circulating Th1, Th17 Cells and Related Cytokines: A Study in Psoriasis Patients in Bangladesh

Op Acc J Bio Sci \& Res 9(1)-2021 
with metabolic diseases in psoriasis patients.

Although the small sample size and a relatively narrow PASI range were insufficient to provide a gross information but data provided here is strongly evident of activation of IL-23/Th17 axis in psoriasis, which is a current topic of interest. Overall, the results of our present study focus the involvement of both Th1 and Th17 cells in psoriasis. The clonal expansion of Th17 and Th1 cells along with associated cytokines and their relation with disease severity and duration underscores their role in local cellular events. Monoclonal antibodies targeting IL-23 are new therapeutic solutions for psoriasis which can potentially stop disease progression as well as remission of cellular events.

\section{Acknowledgments}

The authors thank all the patients and controls for their invaluable cooperation. The authors acknowledge the staffs of the flow cytometry and Immunology laboratory of Microbiology and Immunology Department, for their skillful technical assistance.

\section{Conflicts of Interest}

The authors have no conflicts of interest to declare.

\section{Authorship}

Shirin Tarafder: Conceptualization, Methodology, Validation, Supervision, Writing-reviewing and Editing, Investigation. Sabia Shahin Sultana: Data curation, Investigation, Writing-original draft preparation, Software. All authors: Manuscript writing: All authors: Final approval of manuscript.

Statement of originality: The authors have seen and approved the final version of the manuscript being submitted. The manuscript is the author's original work, hasn't received prior publication and isn't under consideration for publication elsewhere.

Conflict of interest disclosure: The authors declare that they have no competing financial interests or personal relationships that could have appeared to influence the work reported in this paper.

Ethical Statement: The authors are accountable for all aspects of the work in ensuring that questions related to the accuracy or integrity of any part of the work are appropriately investigated and resolved. The study was conducted in accordance with the Declaration of Helsinki (as revised in 2013). This study was approved by institutional review board (IRB). Written informed consent was obtained from the patients for research and publication of this study.

\section{References}

1. Gaffen SL, Jain R, Garg AV, Cua DJ (2014) The IL-23-IL-17 immune axis: from mechanisms to therapeutic testing. Nat Rev Immunol 14(9): 585600.

2. Gerkowicz A, Pietrzak A, Szepietowski JC, Radej S, Chodorowska G (2012) Biochemical markers of psoriasis as a metabolic disease. Folia Histochem Cytobiol 50(2): 155-170.

3. Chen Y, Langrish CL, Mckenzie B, Joyce-Shaikh B, Stumhofer JS, et al. (2006) Anti-IL-23 therapy inhibits multiple inflammatory pathways and ameliorates autoimmune encephalomyelitis. J Clin Invest 116(5): 1317-1326.

4. Hawkes JE, Chan TC, Krueger JG (2017) Psoriasis pathogenesis and the development of novel targeted immune therapies. J Allergy Clin Immunol 140(3): 645-653.

5. Kryczek I, Zhao E, Liu Y, Wang Y, Vatan L, et al. (2011) Human TH17 cells are long-lived effector memory cells. Sci Transl Med 3(104): 104 ra100.

6. Qu N, Xu M, Mizoguchi I, Furusawa JI, Kaneko K, et al. (2013) Pivotal roles of T-helper 17-related cytokines, IL-17, IL-22, and IL-23, in inflammatory diseases. J Immunol Res.

7. Priyadarssini M, Divya Priya D, Indhumathi S, Rajappa M, Chandrashekar L, et al. (2016) Immunophenotyping of T cells in the peripheral circulation in psoriasis. B J Biomed Sci 73(4): 174-179.

8. Langley RG, Ellis CN (2004) Evaluating psoriasis with psoriasis area and severity index, psoriasis global assessment, and lattice system physician's global assessment. J Am Acad Dermatol 51(4): 563-569.

9. Szegedi A, Aleksza M, Gonda A, Irinyi B, Sipka S, et al. (2003) Elevated rate of Thelper1 (TH1) lymphocytes and serum IFN- $\gamma$ levels in psoriatic patients. Immunol Lett 86(3): 277-280.

10. Kagami S, Rizzo HL, Lee JJ, Koguchi Y, Blauvelt A (2010) Circulating Th17, Th22, and Th1 cells are increased in psoriasis. J Invest Dermatol 130(5):1373-1383.

11. Jacob SE, Nassiri M, Kerdel FA, Vincek V (2003) Simultaneous measurement of multiple Th1 and Th2 serum cytokines in psoriasis and correlation with disease severity. Mediators Inflamm 12(5): 309-313.

12. Marinoni B, Ceribelli A, Massarotti MS, Selmi C (2014) The Th17 axis in psoriatic disease: pathogenetic and therapeutic implications. Autoimmun Highlights 5(1): 9-19.

13. Zhang L, Yang XQ, Cheng J, Hui RS, Gao TW (2010) Increased Th17 cells are accompanied by FoxP3+ Treg cell accumulation and correlated with psoriasis disease severity. J Clin Immunol 135(1): 108-117.

14. Bovenschen HJ, Van De Kerkhof PC, Van Erp PE, Woestenenk R, Joosten I, et al. (2011) Foxp3+ regulatory T cells of psoriasis patients easily differentiate into IL-17A-producing cells and are found in lesional skin. J Inves Dermatol 131(9): 1853-1860.

15. Benham H, Norris P, Goodall J, Wechalekar MD, FitzGerald O, et al. (2013) Th17 and Th22 cells in psoriatic arthritis and psoriasis. Arthritis Res Ther 15(5): 1-11.

16. Kyriakou A, Patsatsi A, Vyzantiadis TA, Sotiriadis D (2014) Serum levels of TNF- $\alpha$, IL-12/23p40, and IL-17 in plaque psoriasis and their correlation with disease severity. J Immunol Res.

17. Pietrzak AT, Zalewska A, Chodorowska G, Krasowska D, Michalak-Stoma A, et al. (2008) Cytokines and anticytokines in psoriasis. Clin Chim Acta 394(1-2): 7-21.

18. Arican O, Aral M, Sasmaz S, Ciragil P (2005) Serum levels of TNF- $\alpha$, IFN- $\gamma$, IL-6, IL-8, IL-12, IL-17, and IL-18 in patients with active psoriasis and correlation with disease severity. Mediators Inflamm 2005(5): 273279.

19. Lin VW, Ringold S, Devine EB (2012) Comparison of ustekinumab with other biological agents for the treatment of moderate to severe plaque psoriasis: a Bayesian network meta-analysis. Arch Dermatol 148(12): 1403-1410.

20. Takahashi H, Tsuji H, Hashimoto Y, Ishida-Yamamoto A, Iizuka H (2010) Serum cytokines and growth factor levels in Japanese patients with psoriasis. Clin Exp Dermatol 35(6): 645-649.

21. Oliveira PS, Cardoso PR, Lima EV, Pereira MC, Duarte ALBP, et al. (2015) IL-17A, IL-22, IL-6, and IL-21 serum levels in plaque-type psoriasis in Brazilian patients. Mediators Inflamm.

22. Jacob SE, Nassiri M, Kerdel FA, Singh M (2001) Serum cytokine levels in psoriasis vulgaris. Br J Biomed Sci 58(1): 40-44.

Citation: Sabia Shahin Sultana ${ }^{1}$, Shirin Tarafder ${ }^{\star}$, Ismet Nigar ${ }^{3}$ and ATM Asaduzzaman ${ }^{4}$. Circulating Th1, Th17 Cells and Related Cytokines: A Study in Psoriasis Patients in Bangladesh

Op Acc J Bio Sci \& Res 9(1)-2021 
23. Indhumathi S, Rajappa M, Chandrashekar L, Ananthanarayanan PH, Thappa DM, et al. (2016) Investigation of association of the IL-12B and IL-23R genetic variations with psoriatic risk in a South Indian Tamil cohort. Hum Immunol 77(1): 54-62.

24. Chhabra S, Narang T, Joshi N, Goel S, Sawatkar G, et al. (2016) Circulating T-helper 17 cells and associated cytokines in psoriasis. Clin Exp Dermatol 41(7): 806-810.

25. Ivanov II, McKenzie BS, Zhou L, Tadokoro CE, Lepelley A, et al. (2006) The orphan nuclear receptor ROR $\gamma$ t directs the differentiation program of proinflammatory IL-17+ T helper cells. Cell 126(6): 1121-1133.

26. Markham A (2017) Guselkumab: first global approval. Drugs, 77(13): $1487-1492$
27. McKeage K, Duggan S (2019) Risankizumab: first global approval. Drugs 79(8): 893-900.

28. Ansari AR, Bashyam AM, Feldman SR (2019) Update on Tildrakizumab for Psoriasis. Curr Dermatol Rep 8(4): 319-325.

29. Cheng X, Yu X, Ding YJ, Fu QQ Xie JJ, et al. (2008) The Th17/Treg imbalance in patients with acute coronary syndrome. Clinical immunology 127(1): 89-97.

30. Eid RE, Rao DA, Zhou J, Lo SF, Ranjbaran H, et al. (2009) Interleukin-17 and interferon- $\gamma$ are produced concomitantly by human coronary artery-infiltrating $\mathrm{T}$ cells and act synergistically on vascular smooth muscle cells. Circulation 119(10): 1424-1132. 